\title{
Les relations entre immigrants compatriotes issus de pays en conflit : l'exemple des immigrants bosniens, colombiens et congolais établis à Québec
}

DOI: $10.11567 /$ met.29.3.2 UDK: 314.74:316.7](714:861+497.6+672.4)

Izvorni znanstveni rad Primljeno: 3.7.2013. Prihvaćeno: 6.2.2014.

\section{Stéphanie Arsenault}

École de service social, Université Laval, Canada

stephanie.arsenault@svs.ulaval.ca

\author{
Anaïs Nadeau-Cossette \\ École de Rochebelle, Québec, Canada \\ anais.nadeau-cossette.1@ulaval.ca
}

\section{RÉSUMÉ}

Cet article pose un regard nouveau sur les dynamiques relationnelles existant entre immigrants compatriotes issus de pays en conflit. Inscrite dans une démarche de théorisation enracinée reposant sur les témoignages de 36 immigrants et réfugiés établis dans la ville de Québec, l'étude met en évidence trois scénarios distinguant autant de manières d'appréhender l'apport des compatriotes aux processus d'insertion postmigratoires. Le premier des scénarios observés consiste en la création, par certaines personnes rencontrées, de stratégies d'insertion propices à la contribution collective et massive de compatriotes à celles-ci. Un second scénario consiste plutôt en l'utilisation de stratégies d'insertion impliquant une contribution restreinte de compatriotes alors qu'un troisième et dernier scénario mène à l'exclusion des compatriotes des stratégies d'insertion postmigratoires. Ces scénarios se trouvent par ailleurs modulés par le temps de même que par l'accessibilité des compatriotes et des non-compatriotes.

MOTS-CLÉS : réfugiés, insertion, immigration, Colombie, Bosnie-Herzégovine, République démocratique du Congo, Québec, stratégies d'insertion

\section{INTRODUCTION}

Cet article porte sur les dynamiques relationnelles postmigratoires développées entre immigrants issus d'un même pays connaissant ou ayant connu un conflit armé sur son territoire ou entre ses habitants. Il présente 
les résultats d'une recherche menée dans la ville de Québec auprès d'immigrants et de réfugiés provenant de Bosnie-Herzégovine, de Colombie et de République Démocratique du Congo (RDC). Nous nous sommes donc interrogés sur la place que ces immigrants accordent à leurs compatriotes d'origine dans le vécu qu'ils expérimentent dans leur nouvelle société. La pertinence d'explorer le sujet dans cette ville et avec ces groupes repose, d'une part, sur le fait que la ville soit devenue, au Québec, un acteur de premier plan dans l'accueil d'immigrants et de réfugiés provenant de pays en conflit, notamment par le biais du programme provincial de parrainage et de rétablissement permanent des réfugiés sélectionnés à l'étranger qui prévoit leur installation à l'extérieur de la grande région montréalaise. Elle repose, d'autre part, sur le fait que la ville ait reçu plusieurs centaines de ressortissants bosniens dans les années 1990 et de ressortissants colombiens et congolais dans les années 2000. De 2001 à 2010, la province de Québec dans son ensemble a reçu un total de 440, 860 nouveaux immigrants. Parmi ceux-ci, 60,660 sont venus par la voie du refuge et le tiers d'entre eux se sont vus pris en charge par le programme provincial de parrainage de réfugiés (MICC, 2011a). Alors que le pourcentage occupé par les réfugiés parmi l'ensemble des immigrants reçus au Québec dans la dernière décennie s'établit à 13,75 pour cent, dans la ville de Québec ce pourcentage si situe plutôt à près de 23 pour cent pendant cette même période avec l'accueil d'une moyenne annuelle d'un peu plus de 400 réfugiés au cours de la dernière décennie, soit 3638 réfugiés sur les 15661 immigrants s'étant établis à Québec entre 2000 et 2009 (MICC, 2011b).

\section{Les relations entre immigrants compatriotes issus de pays en conflit dans la littérature scientifique}

Dans la littérature, les auteurs ayant touché aux relations entre immigrants compatriotes dans leur pays de réinstallation ont mis en évidence de nombreux éléments influençant leur nature, leur portée et leur finalité. Parmi ces éléments, les allégeances politiques et idéologiques semblent tantôt constituer une force mobilisatrice importante chez des sous-groupes au sein d'une population provenant d'un même pays (Bermudez, 2007; Bolzman, 2002; Wahlbeck, 1998), tantôt limiter la mobilisation de ces groupes (Guarnizo, Sanchez et Roach, 1999; Moro, 2004). Dans ces derniers cas, la nature politique des relations entre réfugiés de même que la diversité des positions idéologiques face au conflit feraient obstacle à la mobilisation.

Les distinctions ou divisions claniques, ethniques, nationales ou religieuses constituent d'autres éléments pouvant intervenir dans la constitu- 
tion de liens entre compatriotes immigrants issus de pays en conflit. Ces appartenances identitaires deviennent elles aussi tantôt un lieu de support, de solidarité et de sécurité pour certains nouveaux arrivants (CICLG, 2009; Kleist, 2008; Schweitzer, Greenslade et Kagee, 2007), tantôt un lieu où l'effritement des relations sociales entre compatriotes se manifeste (Bermudez, 2011; Bouvier, 2007; Hopkins, 2010; Kleist, 2008; Sorenson, 1990). L'origine, clanique ou autre, des dirigeants de certaines organisations intervenant auprès des exilés peut notamment leur affubler une étiquette partisane, réduisant ainsi l'utilisation des services par des pans de la population visée (Hopkins, 2010).

En regard de l'identité ethnique, Remington Lucken (2010) a pu observer que le degré de cohésion et de solidarité interethnique entre ressortissants d'un même pays différait selon les villes d'établissement. Elle en concluait qu'un groupe fortement hétérogène sur le plan ethnique et religieux, peu pratiquant et s'identifiant faiblement aux repères ethniques apparaît plus uni qu'un groupe multiethnique comptant une majorité plus homogène, religieusement pratiquante et fortement identifiée aux repères ethniques.

Certains auteurs mentionnent également le peu d'intérêts communs entre compatriotes comme cause potentielle de divisions au sein des populations de réfugiés. Les études de groupes de réfugiés établis en Suède (Eastmond, 1998) et au Royaume-Uni (Williams, 2006) révèlent que ces derniers considèrent souvent avoir effectivement peu en commun avec leurs compatriotes, hormis le fait de provenir d'un même pays ou d'une même nation, ce qui les incitait peu à se côtoyer.

Le fait même d'être réinstallés en exil constituerait un autre facteur influençant les relations sociales entre compatriotes. Alors que pour certains l'éloignement du conflit serait bénéfique sur ce plan et contribuerait à calmer les tensions (Bermudez, 2011 ; Bouvier, 2007), pour d'autres la réalité de l'exil deviendrait plutôt une difficulté supplémentaire avec laquelle composer pour leur insertion. Blackwell (1993) estime que les conséquences des conflits sur les individus et leurs réseaux sociaux - comme la rupture des liens sociaux, la méfiance, la peur et la perte du sens de la communauté sont exacerbées par l'exil, ce qui rendrait les relations avec les compatriotes d'autant plus difficiles. La méfiance entre compatriotes est un élément qui revient particulièrement souvent concernant les immigrants et réfugiés colombiens (Arsenault, 2006; Bermudez, 2011; Bouvier, 2007; Charland, 2006; Guarnizo, Sanchez et Roach, 1999) chez qui peu se disent très proches les uns des autres et où plusieurs restent volontairement distants de leurs compatriotes. 
Il semble en outre que des relations intracommunautaires peu harmonieuses peuvent mener à l'exclusion de certains compatriotes qui seront conséquemment plus sujet à une forme d'isolement social. McMichael et Manderson (2004), dans leur recherche sur les Somaliennes établies en Australie, parlent de la rupture des réseaux sociaux engendrée par le conflit dans le pays d'origine qui génère tristesse, anxiété et solitude chez plusieurs. Néanmoins, selon certains auteurs, pour des immigrants issus de pays en conflit, l'isolement de leurs pairs peut être un choix, un mécanisme de défense visant à échapper à l'anxiété et aux souvenirs douloureux qui refont surface lors d'interactions sociales avec des compatriotes (Blackwell, 1993; Bouvier, 2007; Charland, 2006; Miller et al., 2002a; Miller et al., 2002b).

Les stéréotypes ou les préjugés dont sont affublés certains immigrants ou réfugiés dans le pays d'accueil auraient aussi un rôle à jouer sur le désir de se retrouver entre compatriotes ou d'être associés à ces derniers (Bermudez, 2011; Bouvier, 2007; Guarnizo, Sanchez et Roach, 1999). En ce sens, les études de Bermudez (2011) et de Devlin Trew (2010) ont laissé voir que les ressortissants colombiens et irlandais démontraient beaucoup de réserves à être associés à leurs compatriotes affublés de stéréotypes négatifs dans la société d'accueil ou encore propageant des images négatives de leur pays d'origine en terre d'accueil, deux réalités déplaisant à de nombreux répondants.

La taille de la population de réfugiés dans un lieu donné constitue un autre facteur important (Guarnizo, Sanchez et Roach, 1999; Remington Lucken, 2010; Sorenson, 1990; Williams, 2006), mais le sens de l'influence exercée par celle-ci ne fait pas consensus. Remington Lucken (2010) suggère qu'une population importante d'immigrants serait moins unie qu'une peu nombreuse puisque, dans ce dernier cas, les réfugiés tendraient à tisser des liens étroits plutôt qu'à se diviser en sous-groupes. À l'opposé, Guarnizo et ses collaborateurs (1999), ayant étudié deux communautés colombiennes établies dans des villes différentes des États-Unis, avancent quant à eux qu'une population moins importante fournit moins de possibilités de développer des contacts avec des compatriotes et, donc, ne contribue pas à renforcer les liens intracommunautaires.

Plusieurs caractéristiques propres aux immigrants semblent elles aussi avoir un impact sur leurs relations avec leurs compatriotes. Le statut d'immigration constitue l'un de ces facteurs autour duquel les relations sociales des compatriotes immigrants et réfugiés se définissent parfois. Le statut de réfugié fait l'objet de litige au sein de certains groupes d'immigrants et de réfugiés originaires de pays en conflits alors que l'on y construit des caté- 
gorisations de "vrais » et de "faux » réfugiés, ou encore de réfugiés qui " méritent » leur statut et de ceux qui auraient " profité » du système en place, mais dont la vie n’aurait pas été réellement en danger (Lewis, 2010).

Une autre source potentielle de divisions mentionnée de façon importante dans la littérature est la région ou la ville d'origine au pays. Selon plusieurs auteurs, la fragmentation sociale observée chez certains groupes de migrants, notamment chez les Colombiens établis aux États-Unis, au Royaume-Uni et en Espagne, est en partie délimitée par la région d'origine (Bermudez, 2011; Bouvier, 2007; Guarnizo, Sanchez et Roach, 1999). Guarnizo et ses collaborateurs (1999) ajoutent que certains migrants colombiens aux États-Unis limitent leurs interactions personnelles et professionnelles aux individus de leur région d'origine et que des divisions à ce sujet sont observées au sein des associations socioculturelles de cette population. Sorenson (1990) mentionne que l'origine régionale au pays a beaucoup d'importance, empêchant le renforcement d'une identité érythréenne commune.

En plus des tensions intracommunautaires liées à la région d'origine, certains auteurs observent également un clivage entre les immigrants originaires de milieux urbains et ceux originaires de milieux ruraux (Hopkins, 2010; Lewis, 2010; Mahmoud, 2011). Dans l'étude de Lewis, certains répondants affirment ne pas vouloir participer aux rassemblements de la communauté kurde, car ils se sentent différents des autres compatriotes y participant qui sont « Just country people» (2010 : 578). Selon Remington Lucken, il semble même que l'origine rurale ou urbaine soit parfois plus importante que l'origine ethnique : "According to refugee resettlement officials, the rural/ urban divide can create a greater gulf than ethnic affiliation » (2010: 197). En outre, le niveau de scolarisation semble souvent imbriqué avec l'origine rurale ou urbaine des compatriotes, les deux éléments se trouvant fréquemment évoqués conjointement par les auteurs, en relation l'un avec l'autre, comme motifs menant à éviter de côtoyer certains compatriotes (Hopkins, 2010; Lewis, 2010; Remington Lucken, 2010).

La situation économique des nouveaux arrivants et celle de ceux préalablement établis peuvent aussi influencer les relations qu'ils entretiennent avec les membres de leur famille et avec leurs compatriotes (McMichael et Manderson, 2004; Menjívar, 1997). Les répondantes somaliennes de l'étude de McMichael et Manderson (2004) suggèrent que la pauvreté a mené à la diminution de la réciprocité et de la redistribution informelle de ressources entre compatriotes, ce qui a eu comme conséquence d'éroder les réseaux sociaux. 
La classe sociale à laquelle s'identifient ou sont identifiés les immigrants constitue elle aussi une source de fragmentation des réseaux sociaux chez les immigrants étudiés (Bermudez, 2011; Bouvier, 2007; Guarnizo, Sanchez et Roach, 1999; Hopkins, 2010; Lewis, 2010; Remington Lucken, 2010). Selon Bermudez (2011), Bouvier (2007) et Guarnizo et ses collaborateurs (1999), la division selon la classe sociale est particulièrement importante chez les Colombiens. La diaspora colombienne aux États-Unis serait en effet caractérisée par une multitude de petites communautés ayant peu en commun et les organisations se formeraient autour de regroupements d'immigrants des classes jugées supérieures. Les résultats de l'étude de Lewis (2010) démontrent également que les participants, incluant des Kurdes, des Iraniens, des Zimbabwéens et des Somaliens, évitaient les rassemblements de leur communauté, principalement en raison des différences de classes perçues avec leurs compatriotes. Par ailleurs, pour d'autres, l'exil offre la possibilité de développer et d'entretenir des relations sociales qui auraient été impossibles au pays d'origine puisque dans l'étude de Bermudez (2011), des immigrants colombiens affirment avoir pu, après la migration, rencontrer et tisser des liens avec des compatriotes de toutes classes sociales, ce qui semblait impensable au pays.

À la lumière de ces connaissances, des zones grises s'y trouvant et de certaines contradictions apparentes, nous avons voulu poser un nouveau regard sur les dynamiques entre compatriotes établis en exil afin d'améliorer notre compréhension de la place des relations sociales entre compatriotes dans la vie des immigrants issus de pays en conflit ainsi que des facteurs qui influencent les dynamiques relationnelles établies entre eux.

\section{Méthodologie de recherche}

La recherche dont il est ici question vise à mettre en lumière la place que les immigrants et réfugiés provenant d'un même pays connaissant ou ayant connu un conflit armé interne accordent à leurs compatriotes d'origine dans le vécu qu'ils expérimentent dans leur nouvelle société et à mieux décrire les dynamiques relationnelles qui se développement entre ceux-ci. Elle s'inscrit dans une démarche qualitative qui vise à donner la parole aux personnes concernées et à analyser les questions à l'étude à partir du point de vue de ces dernières et du sens qu'elles accordent à leur vécu. Il s'agit donc d'une posture épistémologique qui accorde une importance centrale à la parole des personnes impliquées dans une situation particulière et à leur façon de donner sens à celle-ci (Poupart, 1997). Les outils de collecte de données utilisés, les méthodes d'analyse employées et la façon de rendre les 
résultats dans le cadre de cette étude sont tous de nature qualitative (Pires, 1997). Afin de mieux comprendre les dynamiques à l'œuvre entre les immigrants compatriotes issus de pays connaissant ou ayant connu des conflits internes, nous avons procédé à une démarche principalement inspirée de la théorisation enracinée (Strauss et Corbin, 2004), laquelle nous a menés à la mise en lumière d'un concept central comme outil descriptif des scénarios à l'œuvre. Pour ce faire, nous avons recueilli, traité et analysé les données fournies par 36 participants immigrants issus de trois pays : la Bosnie-Herzégovine (12), la Colombie (12) et la République démocratique du Congo (12). Ces participants, autant d'hommes que de femmes, ont été recrutés par la méthode " boule de neige » et l'échantillon constitué a respecté un souci de diversification (Pires, 1997) quant à l'identité nationale, à l'âge, au statut socioéconomique et à la durée de leur séjour au pays. Une entrevue individuelle semi-dirigée a été réalisée entre 2009 et 2010 avec chacun des participants, lesquelles ont été enregistrées sur support numérique, retranscrites intégralement pour être ensuite codées et analysées avec l'aide du logiciel NVivo8.

Nous avons procédé à trois étapes de codification et d'analyse, soit la codification ouverte, axiale puis sélective telles que décrites par Strauss et Corbin (2004). La codification ouverte, où l'unité de codification utilisée a été chaque idée ou argument énoncés, a permis de mettre en évidence les concepts émergents ainsi que de les décrire. Quarante-huit catégories descriptives ont été identifiées et regroupées en neuf catégories interprétatives que sont : la communication interpersonnelle, les conditions de vie, définition identitaire, expérience du conflit, projet de vie migratoire, relations amicales, le vécu d'insertion et la vie familiale. Pour le processus de codification axiale, nous avons cherché à répondre aux questions de contexte, de conditions causales, de caractéristiques spécifiques de stratégies et de conséquences quant aux dynamiques qui nous intéressent (Laperrière, 1997). La troisième étape de codification sélective nous a finalement permis de faire ressortir une catégorie centrale autour de laquelle l'ensemble des scénarios gravitent ou s'articulent.

Les personnes rencontrées avaient en moyenne 41,5 ans et résidaient à Québec depuis un temps moyen de 7,5 années (13,9 ans chez les Bosniens, 4,5 ans chez les Colombiens et 4,1 ans chez les Congolais). Elles étaient majoritairement mariées (30/36) avec enfants (33/36). Une large majorité s'est installée au Québec par la voie du refuge (26/36), alors que sept sont venus comme immigrants économiques et trois seulement comme membres de la famille parrainés. 


\section{RÉSULTATS}

\section{Les scénarios à l'œuvre}

Les processus de codage ouvert, axial puis sélectif caractéristiques de la théorisation enracinée (Strauss et Corbin, 2004) nous ont permis de mettre en évidence les "stratégies d'insertion postmigratoires " comme axe central autour duquel s'articule l'ensemble des scénarios observés et décrits ciaprès. Nous parlons ici d'insertion au sens large, c'est-à-dire de la place que chacun souhaite prendre au sein de la nouvelle société dans laquelle il évolue désormais, sans par ailleurs avoir pu analyser chacune des dimensions d'un processus d'intégration qui comprend notamment les dimensions économique, politique, familiale et sociale. Consécutivement à l'identification de l'axe central que constitue les stratégies d'insertion, trois scénarios présents chez les immigrants et réfugiés issus de pays en conflit quant aux relations qu'ils développent ou entretiennent entre eux ont émergé. Le premier des scénarios observés (A) consiste en la création de stratégies d'insertion propices à la contribution collective et massive de compatriotes à celles-ci. Un second scénario (B) consiste plutôt en l'utilisation de stratégies d'insertion impliquant une contribution restreinte de compatriotes alors qu'un troisième et dernier scénario $(\mathrm{C})$ mène à l'exclusion des compatriotes des stratégies d'insertion postmigratoires. Comme nous le reprécisons ultérieurement, la question de la construction de ces différentes représentations est une question qui demeure à résoudre au terme de cette étude.

Ces trois scénarios reposent à leur tour, selon le modèle paradigmatique de Strauss et Corbin repris par Laperrière (1997), sur trois hypothèses caractérisant autant de groupes ou catégories de personnes pour qui : (hypothèse A) une représentation positive du rôle potentiel de compatriotes dans l'insertion tend à favoriser leur inclusion dans les stratégies d'insertion; (hypothèse $\mathrm{B}$ ) une représentation mitigée du rôle potentiel de compatriotes dans l'insertion tend à réduire leur inclusion aux stratégies d'insertion et; (hypothèse $\mathrm{C}$ ) une représentation négative du rôle potentiel de compatriotes dans l'insertion tend à exclure leur participation aux stratégies d'insertion. Ces scénarios se trouvent par ailleurs modulés de façon importante par le temps (celui qui passe et celui disponible) de même que par l'accessibilité des compatriotes et des non-compatriotes. Nous revenons plus loin en détail sur ces modulations.

Les résultats démontrent donc une trame allant de l'inclusion maximale des compatriotes, à une extrémité du spectre, à leur évitement à l'autre extrémité, selon qu'ils soient considérés comme une aide potentielle ou 
comme une entrave à l'élaboration des stratégies d'insertion établies par chacun et à l'insertion comme telle poursuivie par chacun. Nous constatons un éventail échelonnant les scénarios tendant vers ces deux extrêmes, sans néanmoins les rejoindre.

Nous désignons les personnes associées au scénario A (6/36) comme étant "initiatrices et inclusives ", les personnes associées au scénario B (12/36) comme étant des « utilisatrices mais mitigées » et celles associées au scénario C (18/36) comme étant « amicales et évasives ». Nous les définissons plus amplement dans les lignes suivantes.

\section{Les initiateurs-inclusifs}

J'ai entrepris quelques petites choses pour les personnes [de mon pays] qui étaient déjà installées ici et qui avaient des petits problèmes dans la famille ou au travail. (...) Je ne connais pas beaucoup, mais c'est de la référence, non? [Comme par exemple] je connais une avocate [de notre pays] qui peut les aider! (...) C'est par ces relations que j'ai dit: Ah, je vais recommencer ma carrière plus officiellement.

Les « initiateurs-inclusifs » du premier scénario sont les participants qui s'appuient le plus sur leurs compatriotes dans la réalisation de leur projet de vie et ils le font délibérément, en choisissant des compatriotes parce qu'ils sont des compatriotes. Les personnes de notre échantillon identifiées à ce groupe ont créé ou souhaitent mettre sur pied des initiatives communautaires ou entrepreneuriales destinées spécifiquement à leurs compatriotes ou à une partie d'entre eux, des initiatives qui permettent notamment le prolongement de l'identité collective à laquelle ils s'associent. C'est le cas notamment d'un homme de Colombie, tel qu'illustré par les propos suivants : «Quand on est arrivés au pays, on a essayé d'être proches des gens qui se connaissaient avant, j'essayais aussi avec quelques autres [compatriotes] de démarrer un club, on a démarré une association. » Il s'agit de personnes dotées d'une identification forte à un collectif associé à leur pays d'origine et qui souhaitent de surcroit maintenir cette identification. " On se connaît. On se rassemble. Il y a le rassemblement quelque part. On fête notre indépendance. C'est toujours comme ça » (femme congolaise).

Ils côtoient ainsi un grand nombre de compatriotes et ont généralement une opinion positive de ceux-ci ou du moins d'une partie de ceux-ci ainsi que du rôle qu'ils peuvent jouer dans leur insertion à la société québécoise. Ils perçoivent de plus leurs compatriotes, ou une partie d'entre eux, comme une communauté unie, ou encore ils émettent le souhait qu'elle le soit. Ils les fréquentent notamment en groupe, c'est-à-dire qu'ils participent à des rassemblements communautaires et sont en ce sens proactifs dans le but de 
rencontrer leurs compatriotes. «On se rassemble, on fait des réunions, des réceptions, des fêtes, on se connaît. Tu ne peux pas trouver trois ou quatre Congolais qui ne me connaissent pas. » (homme congolais).

\section{Les utilisateurs-mitigés}

Ce n'est pas que je ne veux pas avoir des amis colombiens, ni que je préfère non plus, mais ça a tombé comme ça. Moi, si c'était un choix personnel, je dirais que j'irais plus vers les Québécois, étant donné que c'est la meilleure manière de s'intégrer ici, puis de se sortir un peu du ghetto là. Je ne veux pas ça être toujours avec des Colombiens, parce que on reste comme un peu à part de la société, notre but c'est un peu plus de...

Les « utilisateurs-mitigés » ont, comme l'appellation l'indique, une opinion mitigée du rôle que devrait avoir les compatriotes dans leur processus d'insertion. Ils ne sont ni fermés à l'effet d'en côtoyer dans leur parcours d'insertion ni enthousiasmés par cette possibilité, jugeant plutôt que le fait de côtoyer ses pairs compatriotes freine l'apprentissage de la langue et ralentit leur insertion. "Ça rétrécit mon réseau. Ça fait en sorte que je ne puisse pas développer mon réseau ailleurs [mais] on peut échanger sur la culture, on peut échanger sur l'expérience de l'immigration » (homme congolais).

Ce sont des personnes qui entretiennent des liens avec des compatriotes dans la poursuite de leurs stratégies migratoires, mais de façon plus transitoire et davantage comme le fruit du hasard que comme le fruit d'une intention délibérée de se retrouver spécifiquement avec des compatriotes. De plus, ils côtoient surtout des compatriotes avec qui ils ont des points communs liés à leurs priorités et à la voie qu'ils se sont tracée pour s'intégrer. Par ailleurs, contrairement aux « initiateurs-inclusifs », les « utilisateurs-mitigés » ne participent pas, ou alors très peu, aux événements ou aux rassemblements réunissant de nombreux compatriotes. Ils veulent plutôt limiter leur présence et participation à leurs stratégies d'insertion et s'en servent plutôt pour la résolution de besoins particuliers que rencontrent aussi d'autres compatriotes comme les besoins d'apprendre la langue, de se former dans un domaine particulier ou de prier en groupe.

\section{Les amicaux-évasifs}

J'ai dit, faut que tu me présentes des bons Québécois. Pas du monde latino-américain, ça ne m'intéresse pas du tout, je ne suis pas venue pour parler espagnol.

Les " amicaux-évasifs » constituent la catégorie la plus nombreuse de notre échantillon. Cette catégorie regroupe les personnes ayant fait le choix 
de côtoyer un nombre très restreint de compatriotes, notamment parce qu'ils considèrent de prime abord que ces relations nuisent à leur insertion et qu'elles ne se sentent pas d'emblée attirées par eux.

Ils côtoient, en privé seulement, quelques compatriotes avec qui ils ont identifié des points communs forts liés à des intérêts personnels qui transcendent le repère que constitue le pays d'origine. Comme le dit Rose : « Ce n'est pas parce qu'on est tous Congolais qu'on se met tous ensemble. On se met avec qui on a des atomes crochus ». Ils formulent de nombreuses critiques à l'endroit des compatriotes en général et tendent à se dissocier de cette masse pour ne s'identifier qu'à quelques-uns au sein de celle-ci. Ils les côtoient de façon peu fréquente et les évitent intentionnellement lorsque possible, par exemple en n'allant jamais ou alors qu'exceptionnellement aux rassemblements qui leur sont destinés. Les propos d'une Congolaise sont éloquents à ce sujet : "Si les gens ne vont pas aux trucs de Congolais, ça va être comme moi je dis: "Ah je connais la mentalité ", tu vas y aller puis après il va y avoir des dérapages, des manques de respect, là, quelqu'un qui a bu, qui commence à dire n'importe quoi » (femme congolaise). Ils ne recherchent donc généralement pas le contact avec des compatriotes, même s'ils le trouvent parfois au gré de leur cheminement au pays d'accueil.

Par ailleurs, le besoin de sélectionner les personnes qui prendront part à ces stratégies d'insertion ou à la vie privée des participants se retrouve partout dans les témoignages, chez tous les groupes identifiés. Pratiquement toutes les personnes interrogées ont effectivement affirmé faire le choix, d'une manière ou d'une autre, des compatriotes qu'elles vont inclure dans leur vie au Canada et surtout accorder beaucoup d'importance à cette sélection. Ce choix s'effectue selon l'identification d'atomes crochus ou d'intérêts communs comme nous l'avons déjà mentionné, des mots qui reviennent très souvent, surtout chez les Colombiens et les Bosniens, mais aussi chez plusieurs Congolais. Nous détaillons donc ces processus de sélection dans les lignes qui suivent.

\section{Les stratégies de sélection à l'œuvre}

Nous avons pu identifier trois types de sélection-évitement opérés par les participants, soit la sélection ou l'évitement des sujets à aborder avec les compatriotes fréquentés, la sélection ou l'évitement des personnes à côtoyer parmi les compatriotes et la sélection ou l'évitement des lieux ou des événements à fréquenter. Ces trois types de comportements ont été exprimés à maintes reprises par les participants des trois origines et des trois catégories 
identifiées et souvent plus d'un type de sélection est pratiqué par une même personne.

\section{La sélection des sujets}

L'évitement de sujets sensibles constitue un premier type de sélectionévitement observé chez nombre de participants des trois origines et de façon particulièrement récurrente chez les Bosniens : "même aujourd'hui quand je rencontre, à mon travail ou ailleurs, quelqu'un qui est d'autre origine [autre origine nationale du même pays] je fais attention quoi dire " (homme bosnien). Ce sont les sujets politiques ou encore les tabous socioculturels qui sont évités entre compatriotes, de manière à éviter l'apparition de tensions et à maintenir un climat serein entre compatriotes se sachant appartenir à des groupes nationaux, religieux, politiques, idéologiques ou socio-économiques différents. "Parce que la situation politique en Colombie est très compliquée, il y a différents points de vue, tout le monde à sa propre opinion. Moi je n'aime pas parler de ça avec personne, ça peut créer des problèmes » (femme colombienne). Cette stratégie apparaît généralement en amont de celle visant à sélectionner ou à éviter des personnes.

\section{La sélection des personnes}

Dans un second temps, plus de la moitié des personnes ont affirmé choisir de fréquenter des personnes en particulier et en éviter d'autres : « Il faut faire le tri » (femme congolaise). En regard de la sélection-évitement des personnes, quatre grandes catégories de critères ou de facteurs ont été repérées. La première catégorie regroupe toutes les affirmations directement reliées aux motifs du conflit sévissant dans les pays d'origine. Ces distinctions concernent principalement les différences nationales ou ethniques au sein du groupe ainsi que l'importance même accordée à certains éléments identitaires, mais aussi celles concernant le statut migratoire et la position politique ou idéologique en regard du conflit.

Une deuxième catégorie regroupe des affirmations concernant l'appartenance de classe et le niveau de scolarité (les intellectuels versus les non intellectuels), appartenance apparaissant guider les alliances et les affinités susceptibles de laisser place à de l'amitié, à de l'entraide ou à diverses interactions continues : "Dans mes relations sociales je suis plus ouverte aux intellectuels, probablement parce que nous avons des pensées semblables, le comportement, voilà » (femme bosnienne). Dans les cas colombiens et congolais, ces critères différenciatifs, bien que non systématiquement reliés aux conflits respectifs, se retrouvent souvent interreliés avec les positions 
idéologiques sur la réalité conflictuelle de leur pays d'origine.

La troisième catégorie regroupe les affirmations touchant à la provenance urbaine, rurale ou régionale des personnes. Ces affirmations sont apparues chez les Bosniens et les Congolais, mais non chez les Colombiens : «Ce sont les gens qui viennent des campagnes qui détestent et qui ne veulent pas communiquer avec les autres » (homme bosnien). En outre, la réalité du clivage régionale semble particulièrement incisive chez les Congolais chez qui la différenciation entre Kinois (des urbains) et gens de l'Est (des villageois) est omniprésent dans les témoignages ainsi que la dépréciation des uns envers les autres et vice versa : «Il y a toujours des conflits, eux sont de tel endroit, nous sommes de tel endroit, nous on est supérieur aux autres, eux ils sont inférieurs à nous » (femme congolaise). Il s'agit ici aussi d'un facteur tantôt associé au conflit tantôt non.

La dernière catégorie de facteurs différenciatifs évoqués concerne des affirmations touchant une dichotomie établie quant aux attitudes ou au style d'insertion développé. Ces affirmations tendent à opposer de manière ferme ceux qui se plaignent de leur vie ici à ceux qui s'y projettent avec enthousiasme vers l'avenir ainsi que ceux qui veulent du mal à leurs compatriotes en opérant une influence négative sur eux à ceux qui collaborent de bonne foi avec eux. Elles opposent aussi ceux qui sont ici depuis longtemps à ceux appartenant aux vagues récentes d'immigrants, ceux qui s'isolent entre eux à ceux qui se mêlent à la population québécoise ou encore ceux qui ont réussi sur le plan socioéconomique à ceux que l'on dit avoir échoué : «Il y a des gens qui ont échoué. Ils ne veulent pas que toi, quand tu arrives, tu réussisses. Donc, quand je suis arrivé, j'ai eu des amis, mais des amis hypocrites et des vrais amis. Oui, des amis qui ne voulaient pas que j'avance » (homme congolais).

\section{La sélection des lieux et événements}

Nous l'avons évoqué plus haut, la vaste majorité des personnes rencontrées évitent délibérément d'aller dans les lieux ou aux événements qui possèdent une vocation spécifique à regrouper leurs compatriotes alors que huit des douze Bosniens, huit des douze Colombiens et cinq des douze Congolais rencontrés affirment ne pas vouloir fréquenter ces lieux en raison des nombreux problèmes qui s'y produisent, des commérages qui y prolifèrent, de l'intrusion dans leur vie privée que cela occasionne et du manque d'intérêt que ces types de rassemblements leur suscitent. Des propos comme ceux qui suivent sont apparus à maintes reprises : "Ça ne m'intéresse pas. » «Ce n'est pas pour moi. » «J'y suis allé une fois et j'ai dit plus 
jamais! » Ainsi, même parmi les personnes n'excluant pas la fréquentation des lieux communs et même parmi ceux initiant carrément la création de ces lieux, il s'opère néanmoins une sélection de nature à attirer, à inclure ou à rencontrer les compatriotes avec lesquels une affinité est identifiée et à exclure ceux jugés indésirables : "Si on apprend qu'il y a des Rwandais, je ne veux pas être là » (homme congolais).

De façon générale, les participants du groupe des « initiateurs-inclusifs » sont ceux qui utilisent le moins de stratégies de sélection ou d'évitement. Ceux qui le font tentent d'abord d'éviter des sujets avant de couper la relation avec des individus ou d'arrêter de fréquenter des lieux. Un seul participant dit éviter des personnes alors que d'autres fréquentent des compatriotes en faisant simplement attention aux sujets qu'ils abordent. Les individus du groupe A semblent généralement bien s'entendre avec beaucoup de leurs compatriotes, sauf dans des cas qu'ils disent extrêmes, des cas qui peuvent néanmoins exclurent des pans complets de la population concernée. Ils savent par ailleurs également éviter occasionnellement certains lieux qu'ils jugent ne pas concorder avec leurs valeurs ou intérêts.

Chez les participants « utilisateurs-mitigés » la majorité évite surtout les individus et les lieux de rassemblements. Comme ils ne côtoient généralement qu'un nombre restreint de compatriotes et qu'ils le font dans un contexte et pour un besoin précis, on peut penser qu'ils ont peu besoin de recourir à l'évitement des sujets, bien que plusieurs le fassent à l'occasion. Le groupe des « amicaux-évasifs » est celui dont les participants évitent le plus systématiquement les lieux fréquentés spécifiquement par des compatriotes ainsi que des individus en particulier. Ils évitent dans une moindre mesure des sujets sensibles, ce qui peut être dû au peu de compatriotes avec qui ils traitent et à la sélection soigneuse dont ils ont fait l'objet pour fin d'inclusion dans leur vie privée. On peut donc penser que comme la totalité des participants de ce groupe prétend éviter des lieux et des personnes, ils ont moins à éviter des sujets délicats. Il faut également remarquer qu'aucun des participants de ce groupe n'utilise une stratégie de recherche de contact.

\section{Les principaux facteurs modulateurs ou d'influence}

\section{L'influence du conflit}

Nous avons porté notre attention sur le degré d'affectation par le conflit que les différentes personnes rencontrées ont expérimenté et l'avons mis en relief avec les scénarios dans lesquels elles s'inscrivent. Il est d'abord apparu que certaines personnes (16/36) se sont décrites ou ont décrit des 
récits de vie prémigratoires démontrant un fort degré d'affectation par le conflit, c'est-à-dire qu'elles ont subi des sévices physiques, qu'elles ont perdu un ou plusieurs de leurs proches ou encore une grande partie de leurs biens et de leur sécurité. Certaines autres personnes (7/36) présentaient une vision de leur vécu prémigratoire du conflit les concernant laissant plutôt émerger une expérimentation plus modérée de conséquences reliées aux conflits. Ainsi, elles ont vécu en contexte de guerre, ont subi des privations reliées à ce contexte ainsi qu'une réduction importante de leur sécurité et une perturbation négative de leur vie quotidienne, sans par ailleurs subir les drames mentionnés plus haut. Finalement, une troisième catégorie de personnes (11/36) a de leur côté présenté des récits de vie prémigratoire pratiquement exempts de perturbations reliées à la guerre ou aux conflits que connaissait leur pays d'origine. Elles étaient pour plusieurs à l'extérieur du pays pendant le conflit ou encore évoluaient dans un milieu qui les gardaient des fâcheuses conséquences de la guerre. Pour deux personnes, il était impossible d'établie leur degré d'affectation par le conflit.

La majorité des personnes ayant été faiblement affectées par le conflit actif dans leur pays d'origine (8/11) se situent dans le scénario $C$ des « amicales-évasives », soit celui visant l'exclusion des compatriotes des stratégies d'insertion alors que les trois autres se situent dans le scénario mitoyen (B) qui envisage leur participation de façon mitigée. Elles sont en contrepartie absentes du scénario A qui implique l'inclusion maximale des compatriotes aux stratégies d'insertion. Nous pouvons aussi observer que cinq des sept personnes moyennement affectées par le conflit se situent dans le scénario $C$ alors que les deux autres se répartissent entre les deux premiers scénarios. C'est donc dire que la majorité des personnes faiblement ou moyennement affectées par le conflit dans leur pays d'origine (13/18) sont des personnes qui ne souhaitent pas ou n'ont pas à inclure leurs compatriotes à leurs stratégies d'insertion, même bien au contraire, elles préfèrent les en exclure.

Contrairement aux deux premières catégories qui se retrouvent concentrées principalement dans le scénario $C$, les personnes fortement affectées par le conflit au pays d'origine se retrouvent réparties à part égale dans les trois scénarios. Ainsi, un nombre semblable d'entre eux favorise le recours maximal aux compatriotes pour s'intégrer (5/16), se sent indécis quant à cette avenue (6/16) ou encore se sent carrément dérangé par la participation de leurs compatriotes aux stratégies d'insertion (5/16). Il semble ici pertinent de relever le fait que les cinq personnes fortement affectées par le conflit situées dans le scénario $C$ sont en fait celles se situant à l'extrémité de cette catégorie et donc du spectre, c'est-à-dire qu'elles excluent très fortement leurs 
compatriotes de leurs stratégies d'insertion, qu'elles comptent sur un cercle particulièrement restreint et sélectif d'amis et qu'elles se distancient souvent très fortement de leur pays d'origine en parlant par exemple «d'ex-pays d'origine » et « d'ex-compatriotes » (femme bosnienne). Il apparaît ainsi que les deux extrémités du spectre constitué par notre échantillon soient occupées par des personnes ayant été fortement affectées par la guerre dans leur pays d'origine.

\section{Le désir d'insertion à la société québécoise et ses limites}

Le désir de s'intégrer à la société québécoise et d'être en liens avec des Québécois d'origines variées prime sur celui de maintenir l'univers socioculturel d'origine. C'est ce qui ressort avec force de presque tous les récits, peu importe le scénario d'insertion emprunté. Face à ce portrait, il est notamment apparu que l'inclusion maximale de compatriotes aux principales stratégies d'insertion n'entrave guère le processus d'enracinement et la construction du sentiment d'être chez soi au Québec, comme l'illustrent les propos de l'un des « initiateur-inclusif » : « Je suis complètement assimilé! J'ai n'ai aucun problème, je me sens mieux à Québec, moi personnellement, qu'aujourd'hui dans ma ville natale. Je me sens plus Canadien que Bosniaque » (homme bosnien).

Par ailleurs, l'ouverture ressentie par les nouveaux arrivants de la part des membres de la société hôte n'est pas toujours à la hauteur des attentes entretenues et ce manque devient parfois une entrave à la construction de liens significatifs entre les immigrants et des membres de la population d'accueil. Plusieurs témoignages ont en ce sens mis en lumière des scénarios dans lesquels les personnes concernées semblaient se trouver par dépit, c'està-dire à défaut de pouvoir ou de savoir comment rencontrer des membres de la population québécoise et d'établir des relations de confiance avec eux. Elles étaient par ailleurs d'emblée mises en contact étroit avec de nombreux compatriotes, le plus souvent dans les classes d'étude de la langue française ou dans les organisations destinées à accompagner leurs premiers pas au Québec. De cette façon, certaines personnes présentaient un nombre et une intensité de fréquentations avec des compatriotes largement supérieurs à ce qu'ils souhaitaient selon la représentation idéale qu'ils adoptaient à l'égard du rôle que devraient jouer leurs compatriotes dans leur vie postmigratoire. Un colombien situé dans le scénario des « initiateurs-inclusifs » illustre de façon exemplaire cette réalité : «On ne peut pas rester juste avec des Colombiens. Une fois que tu commences à parler un peu, à te débrouiller en français, à parler un petit peu plus, essaye de rencontrer d'autres personnes, 
essaye de vraiment [te demander] c'est quoi qui t'intéresse ici? En tout cas, moi-même je me fais cette critique. Avec toute l'ouverture qu'on a dans ma famille, tout ça, malheureusement le pourcentage de Québécois dans nos vies ça reste plus petit que $5 \%$. Je pense que dans mon cas particulier, ça dépend beaucoup des autres. Parce qu'on essaye, mais...».

\section{Le temps}

Tout comme l'importance accordée à la sélection des compatriotes à inclure dans leur vie postmigratoire, la notion de temps est également omniprésente dans les témoignages recueillis. Le passage du temps semble en effet avoir une influence sur la réduction des besoins auxquels peuvent répondre les compatriotes, mais aussi sur la disponibilité des personnes à l'égard des relations sociales et privées avec des compatriotes : "Au début, c'était important, mais c'est comme ça, maintenant c'est moins important [avoir des amis compatriotes]. (...) Ici, le travail prend beaucoup de place. Beaucoup, beaucoup de place » (femme bosnienne). Ainsi, au fil des ans, de plus en plus de personnes travaillent, fondent une famille et manquent de temps pour entretenir une vie sociale externe à la vie familiale. Par ailleurs, le temps apparaît également exercer une influence positive sur la connaissance mutuelle des personnes et de l'environnement et donc sur la capacité de choisir les «bonnes » personnes et les «bons » lieux de fréquentation à inclure dans sa vie postmigratoire. Dans un tel contexte, il semble que les tensions diminuent avec le temps entre compatriotes, mais que la raison en soit le fait que chacun ait pu faire ses choix, et non la disparition des blessures ou des animosités entre individus ou entre sous-groupes. Une femme bosnienne l'exprime en ces mots : «Alors maintenant on sait c'est qui, où c'est la place de qui, tout le monde s'est placés. Il n'y a plus de nouveaux pour déséquilibrer ça. (...) On se connaît déjà, on sait que dans cette ville il y a peut-être mille âmes qui sont arrivées de l'Ex-Yougoslavie, puis on sait qui est qui ». En somme, chez pratiquement toutes les personnes rencontrées le nombre et la fréquence des rencontres avec les compatriotes diminuent avec le temps, une réalité qui est particulièrement patente chez les Bosniens établis à Québec depuis plus longtemps que les ressortissants des deux autres groupes étudiés.

\section{CONCLUSION}

De ces analyses, plusieurs observations nous apparaissent particulièrement importantes à retenir. Comme plusieurs de recherches antérieures, no- 
tre étude met en évidence plusieurs bienfaits ressentis par des immigrants issus de pays en conflit tirés des liens établis entre compatriotes. Mais elle fait elle aussi ressortir les limites et les écueils reliés à ces relations et nous permet de mieux comprendre différents scénarios à l'œuvre.

À la suite d'un processus inspiré de la théorisation enracinée, les résultats de notre étude démontrent une trame théorique s'articulant autour des stratégies d'insertion élaborées par les répondants et de leurs représentations variables de la place que devraient y occuper leurs compatriotes. Cette trame se déploie sur un éventail de possibilités allant de l'inclusion maximale des compatriotes aux stratégies d'insertion à leur évitement quasicomplet, selon qu'ils soient considérés comme une aide potentielle positive ou au contraire comme une entrave à l'insertion poursuivie par chacun.

Nous avons donc observé trois scénarios cristallisés par les appellations « d'initiateurs-inclusifs », «d'utilisateurs-mitigés » et « d'amicaux-évasifs ». Nous avons également établis que ces scénarios se trouvent modulés de façon importante par le temps de même que par l'accessibilité et l'ouverture des Québécois d'origines diverses. Presque tous ont en effet moins de relations, de toute nature, avec leurs compatriotes au moment de l'entrevue que lors des mois ou des années suivant leur arrivée au Québec. De plus, une majorité, située surtout dans les scénarios $B$ et $C$, regrette le fait qu'il lui soit si difficile d'établir des liens de confiance avec des Québécois d'origines diverses, et particulièrement avec les Québécois " de souche », raison pour laquelle ils recourent, en quelque sorte par dépit, surtout à des compatriotes connus d'entrée de jeu lors du voyage en avion, de la période d'apprentissage de la langue française, de la fréquentation des organismes d'accueil ou des églises.

Nous avons finalement identifiés différentes formes de sélection traversant tous les scénarios observés et toutes les origines rencontrées. L'importance de la sélection et du pouvoir de choisir les personnes à inclure dans la vie postmigratoire apparaît indiscutable, telle une forme fondamentale de prise de pouvoir sur sa vie. Cette sélection repose tantôt sur des facteurs reliés au conflit en cause dans les pays d'origine, tantôt sur des facteurs d'un tout autre ordre. En effet, si l'étude des comportements et des attitudes des ressortissants de pays en conflit établis au Québec met en évidence les répercussions de ces conflits sur les dynamiques développées entre eux en exil, il appert également que de nombreux autres facteurs, n'ayant rien à voir avec la guerre, guident également les alliances et les inimitiés possibles. En ce sens, il nous apparaît que les gens issus de pays en conflit jaugeront leurs compatriotes d'origine à partir d'un plus grand nombre de 
facteurs potentiellement unificateurs ou problématiques, mais que les immigrants de toute provenance et de tout statut migratoire sont également susceptibles de soupeser minutieusement l'inclusion de leurs compatriotes dans leur vie postmigratoire.

À cet égard, nous regardons avec intérêt le fait que les personnes présentant les expressions les plus extrêmes de notre spectre, c'est-à-dire celles incluant le plus largement leurs compatriotes aux différentes sphères de leur vie comme celles les excluant le plus rigoureusement s'avèrent être les personnes les plus durement et directement touchées par le conflit dans leur pays d'origine. Il semble donc que cet élément expérientiel, combiné à d'autres éléments contextuels, poussent certaines personnes vers la création d'alliances importantes avec des compatriotes choisis et d'autres vers le repli complet face à ceux-ci, des réactions donc opposées. Ces observations suscitent à leur tour d'importantes questions auxquelles nous n'avons encore pu répondre concernant les processus de construction des différentes représentations existantes quant aux rôles et à la place que devraient prendre les compatriotes dans la vie postmigratoire et dans les processus d'insertion, qui demandent à être mieux compris, ainsi que concernant les conditions d'accueil propices à faciliter ou à obstruer les rencontres et les alliances entre les nouveaux venus et la société locale déjà en place.

L'origine n'apparaît pas constituer un facteur suffisant sur lequel reposer les alliances et leurs affinités. Au contraire, l'importance de l'insertion dans la société québécoise et du désir d'être avec des gens qui nous ressemble, au-delà de l'origine nationale ou géographique, prime. Dans cette même ligne, la diversité intra-compatriote est souvent associée à différentes formes de tensions potentielles ou de repli alors que la diversité extra-compatriote est perçue plus positivement et contribuant à l'intégration à long terme recherchée.

Des questions se posent également en regard de l'observation voulant que les gens plus faiblement touchés par la guerre au pays d'origine ne souhaitent que rarement se mêler étroitement à leurs compatriotes en contexte postmigratoire, des gens qui ont, eux, été majoritairement affectés par la guerre. Nous avançons deux hypothèses explicatives à cet égard. Il apparaît plausible que ces personnes, étant venues pour la plupart pour des raisons socio-économiques ou conjugales, aient davantage développé leur intérêt face au défi de poursuivre la construction de leur vie dans un nouvel environnement socio-économique, une nouvelle langue et une nouvelle culture que leurs compatriotes réfugiés ayant fui pour des raisons de persécutions, et qu'elles soient donc davantage préparées à une rupture d'avec leur pays 
d'origine. Il apparaît également plausible que leurs réticences à l'égard de leurs compatriotes soient accentuées par l'inconnu que représente le vécu de la guerre et la peur que ce vécu expérimenté par leurs compatriotes vienne perturber leur processus d'insertion à la société québécoise.

Retenons donc que les relations entre immigrants compatriotes issus de pays en conflits sont porteuses de défis, qu'elles ne vont pas de soi et que certains les souhaitent alors que d'autres les refusent plutôt. De toute évidence, plus nombreuses sont les personnes désireuses de contacts significatifs avec des personnes partageant des intérêts communs issues de la société d'accueil, ce qui est largement perçu comme synonyme d'une intégration réussie. La référence au pays d'origine n'est donc pas d'une importance et d'une pertinence capitale pour une majorité des gens rencontrés. Il apparaît plus que jamais nécessaire de rendre possibles et accessibles les rencontres de diverses natures entre la population arrivante concernée et celle déjà établie, notamment par le biais d'une insertion socio-économique plus rapide et par toutes sortes d'initiatives impliquant d'emblée la population nouvelle arrivante et celles dites « de souche » et immigrante de toutes origines.

\section{RÉFÉRENCES}

Arsenault, S. (2006). Transnacionalismo: el caso de los refugiados colombianos en Quebec. Grenade: Université de Grenade, Département d'anthropologie sociale.

Barnes, D. M. et Aguilar, R. (2007). Community Social Support for Cuban Refugees in Texas, Qualitative Health Research, 17 (2): 225-237, doi: 10.1177/1049732306297756.

Bermudez, A. (2011). The "Diaspora Politics" of Colombian Migrants in the UK and Spain, International Migration, 49 (3): 125-143, doi: 10.1111/j.1468-2435.2011.00689.x.

Blackwell, R. (1993). Disruption and Reconstitution of Family, Network and Community System Following Torture, Organised Violence and Exile, in: J. P. Wilson et B. Raphael (éds). International Handbook of Traumatic Stress Syndromes. New York: Plenum Press, 733-741.

Bolzman, C. (2002). De l'exil à la diaspora: l'exemple de la migration chilienne, Autrepart, 22: 91-107.

Bouvier, V. M. (2007). A Reluctant Diaspora? The Case of Colombia, in: H. Smith et P. Stares (éds). Diasporas in conflict: Peace-Makers or Peace-Wreckers. Tokyo: United Nations University Press, 129-152.

Charland, M. (2006). La confiance au cour de l'exil: Récit de réfugiés colombiens (mémoire de maîtrise). Québec: Université Laval.

CICLG - CHANGE INSTITUTE, COMMUNITIES AND LOCAL GOVERNMENT (2009). The Somali Muslim Community in England: Understanding Muslim Ethnic Communities. London : Communities and Local Government. 
Devlin Trew, J. (2010). Reluctant Diaspora of Northern Ireland: Migrant Narratives of Home, Conflict, Differences, Journal of Ethnic and Migration Studies, 36 (4): 541-560, doi: 10.1080/13691830903520424 .

Doraï, M. K. (2003). Palestinian Emigration from Lebanon to Northern Europe: Refugees, Networks, and Transnational Practices, Refuge: Canada's Journal on Refugees, 21 (2): 23-31.

Eastmond, M. (1998). Nationalist Discourses and the Construction of Difference: Bosnian Muslim Refugees in Sweden, Journal of Refugee Studies, 11 (2): 161-181, doi:10.1093/ jrs/11.2.161.

Guarnizo, L. E., Sanchez, A. I. et Roach, E. (1999). Mistrust, Fragmented Solidarity and Transnational Migration: Colombians in New-York City and Los Angeles, Ethnic and Racial Studies, 22 (2): 367-396, doi: 10.1080/014198799329521.

Hopkins, G. (2010). A Changing Sense of Somaliness: Somali Women in London and Toronto, Gender, Place and Culture, 17 (4): 519-538, doi: 10.1080/0966369X.2010.485846.

Kleist, N. (2008). Mobilising 'The Diaspora': Somali Transnational Political Engagement, Journal of Ethnic and Migration Studies, 34 (2): 307-323, doi: 10.1080/13691830701823855.

Laperrière, A. (1997). La théorisation enracinée (grounded theory): démarche analytique et comparaison avec d'autres approches apparentées, in: J. Poupart et al. (éds). La recherche qualitative : Enjeux épistémologiques et méthodologiques. Montréal: Gaëtan Morin éditeur, 309-332.

Lewis, H. (2010). Community Moments: Integration and Transnationalism at Refugee Parties and Events, Journal of Refugee Studies, 23 (4): 571-588, doi:10.1093/jrs/feq037.

Mahmoud, H. W. (2011). “Conflict Defines Origin": Identity Transformations of Sudanese Refugees in Cairo, Conflict Resolution Quarterly, 28 (3): 263-289, doi: 10.1002/crq.20023.

McMichael, C. et Manderson, L. (2004). Somali Women and Well-Being: Social Networks and Social Capital among Immigrant Women in Australia, Human Organization, 63, 88-99.

Menjívar, C. (1997). Immigrant Kinship Networks and the Impact of the Receiving Context: Salvadorans in San Francisco in the Early 1990s, Social Problems, 44 (1): 104-123.

MICC - MINISTÈRE DE L'IMMIGRATION ET DES COMMUNAUTÉS CULTURELLES (2011a). Tableaux sur l'immigration permanente au Québec, 2006-2010. Québec: Gouvernement du Québec.

MICC - MINISTÈRE DE L'IMMIGRATION ET DES COMMUNAUTÉS CULTURELLES (2011b). Portraits régionaux 2000-2009. Caractéristiques des immigrants établis au Québec et dans les régions en 2011. Québec: Gouvernement du Québec.

Miller, K. E, Muzurovic, J., Worthington, G. J., Tipping, S. et Goldman, A. (2002a). Bosnian Refugees and the Stressors of Exile: A Narrative Study, American Journal of Orthopsychiatry, 72 (3): 341-354, doi: 10.1037/0002-9432.72.3.341.

Miller, K. E., Weine, S. M., Ramic, A., Brkic, N., Djuric Bjedic, Z., Smajkic, A., Boskailo, E. et Worthington, G. (2002b). The Relative Contribution of War Experiences and Exile-Related Stressors to Levels of Psychological Distress among Bosnian Refugees, Journal of Traumatic Stress, 15 (5): 377-387, doi: 10.1023/A:1020181124118.

Moro, L. N. (2004). Interethnic Relations in Exile: The Politics of Ethnicity among Sudanese Refugees in Uganda and Egypt, Journal of Refugee Studies, 17 (4): 420-436, doi:10.1093/jrs/17.4.420. 
Pires, A.P. (1997). Échantillon et recherche qualitative : essai théorique et méthodologique, in: J. Poupart et al. (éds). La recherche qualitative : Enjeux épistémologiques et méthodologiques. Montréal : Gaëtan Morin éditeur, 113-172.

Poupart, J. (1997). L'entretien de type qualitatif : considérations épistémologiques, théoriques et méthodologiques, in: Jean Poupart et al. (éds). La recherche qualitative : Enjeux épistémologiques et méthodologiques. Montréal: Gaëtan Morin éditeur, 174-209.

Remington Lucken, K. (2010). Identity Matters: Bosnian Identity Maintenance in a PostMigration Setting (thèse de doctorat). Boston: Boston University.

Schweitzer, R., Greenslade, J. et Kagee, A. (2007). Coping and Resilience in Refugees from the Sudan: A Narrative Account, Australian and New Zealand Journal of Psychiatry, 41 (3): 282-288, doi: 10.1080/00048670601172780.

Sorenson, J. (1990). Opposition, Exile and Identity: The Eritrean Case, Journal of Refugee Studies, 3 (4): 298-319.

Strauss, A. et Corbin, J. (2004). Les fondements de la recherche qualitative. Techniques et procédures de développement de la théorie enracinée. Fribourg: Academic Press Fribourg.

Wahlbeck, Ö. (1998). Community Work and Exile Politics: Kurdish Refugee Associations in London, Journal of Refugee Studies, 11 (3): 215-230, doi:10.1093/jrs/11.3.215.

Williams, L. (2006). Social Networks of Refugees in the United Kingdom: Tradition, Tactics and New Community Spaces, Journal of Ethnic and Migration Studies, 32 (5): 865-879, doi: 10.1080/13691830600704446. 


\title{
Odnosi između imigranata sunarodnjaka iz zemlje u sukobu: primjer bosanskih, kolumbijskih i kongolskih imigranata nastanjenih u Quebecu
}

\author{
Stéphanie Arsenault, Anaïs Nadeau-Cossette
}

\begin{abstract}
SAŽETAK
U radu se daje novi pogled na odnose imigranata sunarodnjaka iz zemlje u sukobu. Primjenom utemeljene teorije (grounded theory) na svjedočenja 36 imigranata i izbjeglica nastanjenih u Quebecu u istraživanju se ističu tri scenarija razlikovanja triju načina razumijevanja doprinosa sunarodnjaka u postmigracijskom procesu integracije. U okviru prvoga promatranoga scenarija neki ispitanici stvaraju integracijske strategije kojima se promiče kolektivni i masovni doprinos sunarodnjaka. Drugi scenarij zapravo karakterizira uporaba integracijskih strategija koja uključuje ograničeni doprinos sunarodnjaka, dok treći dovodi do isključivanja sunarodnjaka iz postmigracijskih integracijskih strategija. Ti se scenariji usklađuju ovisno o vremenu i dostupnosti sunarodnjaka i nesunarodnjaka.
\end{abstract}

KLJUČNE RIJEČI: izbjeglice, integracija, imigracija, Kolumbija, Bosna i Hercegovina, Demokratska Republika Kongo, Quebec, integracijske strategije 


\title{
The Relationship between Immigrants Compatriots from Countries in Conflict: the Example of Bosnian, Colombian and Congolese Immigrants Established in Quebec City
}

\author{
Stéphanie Arsenault, Anaïs Nadeau-Cossette
}

\begin{abstract}
SUMMARY
This article presents a new look on the relations established between immigrants coming from a same country in conflict. Based on the grounded theory applied on testimonies of 36 immigrants and refugees established in Quebec City, this study highlights three scenarios distinguishing three ways to understand the contribution of the compatriots in the post-migration integration process. The first observed scenario is the creation, by some respondents, of integration strategies including numerous compatriots. A second scenario is rather characterised by the use of integration strategies involving a restricted contribution of compatriots while a third scenario leads to exclude the compatriots of the post-migration integration strategies. These scenarios are also modulated by time as well as by the accessibility of compatriots and non-compatriots.
\end{abstract}

KEY WORDS: refugees, integration, immigration, Colombia, Bosnia and Herzegovina, Democratic Republic of Congo, Quebec, integration strategies 